\title{
Integrating context of tumor biology and vaccine design to shape multidimensional immunotherapies
}

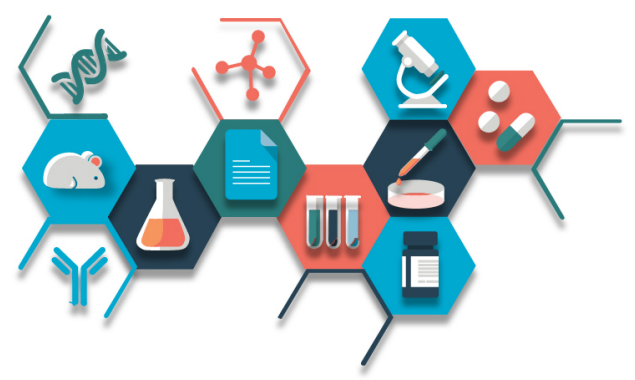

\author{
Nathaniel S Wang ${ }^{*, 1} \&$ Christian J Maine ${ }^{1}$ \\ ${ }^{1}$ Janssen Research \& Development, La Jolla, CA 92121, USA \\ *Author for correspondence: nathanielwang@gmail.com
}

\begin{abstract}
Advances in cancer therapy have offered great promise but only modest clinical benefits as monotherapies to date. Patients usually respond well to therapies targeted at specific mutations, but only for a short time. Conversely, immunotherapies help fewer patients, but increase survival. Combination therapies, which could offer the best of both worlds, are currently limited by substantial toxicity. While recent advances in genomics and proteomics have yielded an unprecedented depth of enabling datasets, it has also shifted the focus toward in silico predictions. Designing the next wave of multidimensional immunotherapies will require leveraging this knowledge while providing a renewed emphasis on tumor biology and vaccine design. This includes careful selection of tumor clinical stage in the context of preexisting tumor microenvironments, target antigen and technology platform selections to maximize their effect, and treatment staging. Here, we review strategies on how to approach an increasingly complex landscape of immunotherapeutic agents for use in combination therapies.
\end{abstract}

Lay abstract: In cancer patients, the use of a single drug that stimulates the immune system has had promising initial results, but still could be improved. The use of multiple drugs together has been challenging for two reasons. First, the negative side effects for the patient are often intolerable. Second, there can be too many choices of drugs to combine. A recent explosion of data has created a heavy reliance on computers to sort through the data. To find a tractable way to create new, rational and effective combinations of drugs for patients, we must account for the underlying biology.

First draft submitted: 11 October 2019; Accepted for publication: 12 December 2019; Published online: 5 February 2020

Current approaches leveraging oncology vaccines in combination therapies have shifted toward neoantigens with ambitious goals of targeting a large proportion of patients in several cancer types. In the process of integrating bioinformatic, genomic and proteomic tools, biology has increasingly taken a backseat to in silico predictions. Clinically validated tumor-associated antigen (TAA) targets have had diminishing interest due to their failures as monotherapies and are becoming undervalued as components of combination therapies. To successfully develop drugs in this space, there will need to be a more realistic set of treatment goals, combined with a renewed focus on the biology and staging of combination therapies.

To develop a new class of multidimensional therapies, the following factors need to be addressed. First, careful selection of the patient population, inclusive of the clinical stage of their cancers, need to be incorporated into treatment regimens to account for implications of tumor evolution on success rates. Next, patients may need to be preconditioned to create a more permissive environment for the de novo generation of tumor-specific responses. For vaccine-based approaches, selection of antigens taking into account multiple aspects of biology allowing for both therapeutic and prophylactic immunizations would be ideal. These must be delivered by technology platforms that generate the right magnitude, quality and balance of $\mathrm{T}$ cell and antibody responses. Finally, existing immunotherapeutic agents that help maintain de novo generated responses need to be leveraged to prevent either dysfunction or exhaustion. Importantly, these considerations should be integrated at the beginning of the design and testing of therapeutic regimens. In the next sections, we will address how to tackle each of these issues to generate more rational combinations. 


\section{Selection of clinical stage for targeting}

Clonal evolution occurs in tumors as individual cells are somatically mutagenized. These somatic mutations can be categorized as driver or passenger mutations, the former resulting in pro-oncogenic alterations that confer selective advantages [1-3]. Drivers can be further categorized into gain-of-function or loss-of-function mutations. Gain-offunction mutations often occur at the same portion of a gene, termed mutational hotspots, which are related to altering gene function to promote cancer, such as at the active sites of proteins [4]. Alternatively, loss-of-function mutations can occur in any portion of a gene and, in addition to single-nucleotide polymorphisms, often involve frameshifts, truncations or other disruptive mutations [5]. In contrast, passenger mutations may occur in any part of the gene or noncoding regions and do not directly contribute to oncogenesis [2].

Tumors progress through multiple stages over the course of clinical treatments [6]. The first phase is a response to the treatment, characterized by reduction in tumor growth. This is followed by a tolerance phase, where resistant clones arise and the tumors may maintain a steady state of growth. In most cases, this ultimately ends in a resistance phase, where individual resistant clones overtake the tumor in response to selective pressure, resulting in renewed tumor growth [7]. By example, the response to immune recognition of the tumor (driven by steady state immunity or immunotherapy-induced immunity) is immunoediting, where mutations recognized by the immune system are removed at the DNA level in individual clones to evade immune-mediated clearance [8-11]. Similarly, targeted therapeutics can yield modifications within mutational hotspots in genes that confer a resistant phenotype. In a recent clinical study, it was observed that $60 \%$ of non-small-cell lung cancer patients progressing or relapsing on erlotinib/gefitinib develop the EGFR T790M mutation, which confers resistance to the treatment [12].

Ideally, focus needs to be on clinical stages of tumors that allow for off-the-shelf vaccines, which have the advantage of being cost-effective and rapidly deployable to patients. This excludes approaches primarily leveraging passenger mutations, which are rapidly immunoedited from tumors since they are dispensable to tumor function. Current treatments are unlikely to generate enough antigen-specific $\mathrm{T}$ cells that will home to the tumor and eliminate all mutation-containing tumor cells prior to onset of immunoediting. However, this then necessitates finding targets that are stably expressed in tumor cells. Stable expression is classically thought to be restricted to driver mutations that initiate tumorigenesis, and as such represent a pool of neoantigens that can be shared among patients, tumor types and stages (termed 'shared neoantigens'). This concept has been appreciated since before the idea of personalized neoantigen prediction and has been used in clinical trials from the mid 1990s [13].

In reality, tumors go through multiple evolutionary stages as they progress from preneoplastic lesions to fully metastatic [14], which complicates the concept of stable expression. For driver mutations, immunoediting can be more complex. Driver mutations can be separated into three types: those present at all stages of the tumor (persistent drivers); those arising at certain stages, but subsequently become dispensable (stage-specific drivers); and some whose loss are coincident with the rise of other mutations (compensatory drivers) (Figure 1) [15,16]. The first type may include mutations in critical tumor suppressor genes, such as TRP53 or P53 [15]. While some specific inactivating mutations may be variable, a mutation in such a gene is likely to occur in certain cancers at all stages. In contrast, the second type of driver mutations may be present at some stages, but not others. EP300 mutations are often present in Stage 2 and Stage 3 of cervical squamous cell carcinoma and endocervical adenocarcinoma, but not in Stages 1 or 4 [15]. Similarly, another study found the number of late-arising driver mutations was equal to or higher than early arising drivers in $44 \%$ of patients with metastatic disease [16]. It may therefore be easier for tumors to immunoedit any immunogenic epitopes in proteins that have these types of kinetics, but only at stages where the protein is dispensable. Finally, the third type of driver can be immunoedited, but only if a complementary mutation occurs. For example, activating mutations in KRAS, which is downstream of EGFR in their signaling pathway, would render mutations in EGFR dispensable [17]. In general, driver mutations being less constrained than conventionally assumed likely contributes to observed tumor heterogeneity. This is especially true when comparing primary tumor cells versus metastases. For example, mutations required for metastasis and survival at new distal microenvironments likely differ from mutations allowing survival within the microenvironment at the origination site. Focus should therefore be on specific clinical stages where targets are stably expressed and have mitigation strategies in place for targets that have a high likelihood of developing compensatory mutations. These mitigation strategies could involve co-administered vaccines, or off-the-shelf vaccines that could be deployed once the compensatory mutations are identified. Once the appropriate stage has been identified, the focus should shift toward ensuring that patients are appropriately preconditioned to respond to the combination therapy. 
(A)

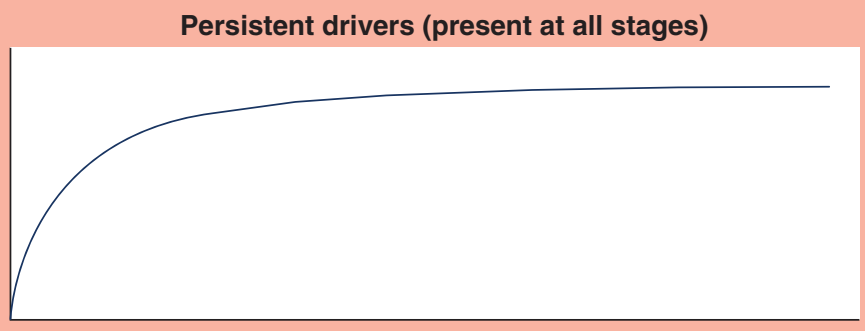

(B) Stage specific drivers (may oscillate at different stages)

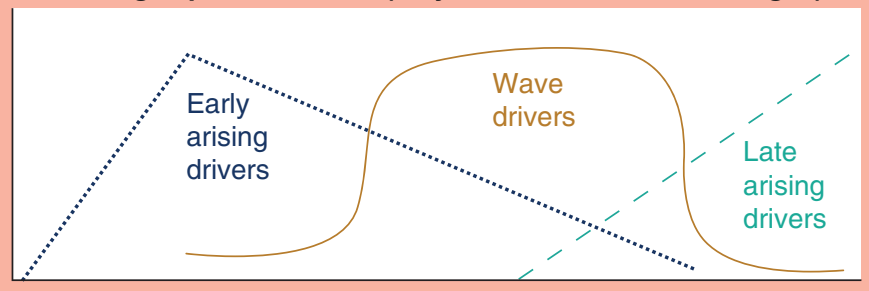

(C) Compensatory drivers (vanish after compensatory mutation occurs)

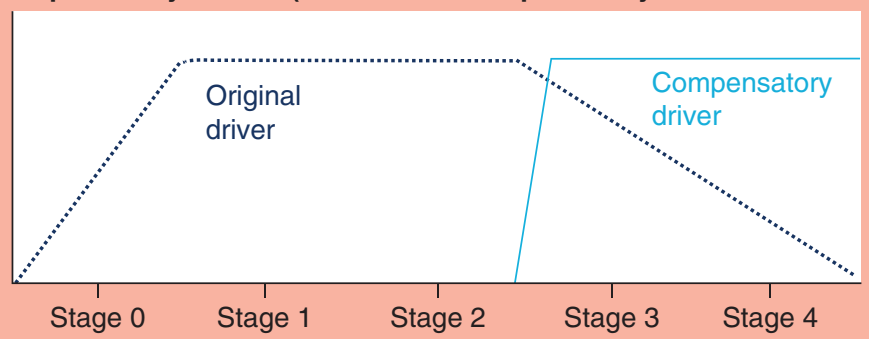

Figure 1. Types of driver mutations over tumor stages. (A) Persistent drivers would be genes whose mutated functions would be required at all stages of the tumor. Examples of this would be tumor suppressor genes. For loss-of-function mutations, the specific inactivating mutation might change over time. (B) Stage specific drivers may have expression that oscillates over time, being present at certain stages but dispensable at others. Examples include EPI300, which is present at Stage 2 and 3 of cervical squamous cell carcinoma and endocervical adenocarcinoma but not at Stage 1 or 4 (red line). Additional examples are late arising drivers (green line), or early arising drivers (blue) that are subsequently lost. (C) Drivers (dotted blue line) may be critical until a compensatory mutation in part of the same or redundant cellular pathway occurs (solid blue line). Examples include activating mutations in KRAS, which would render activating mutations in EGFR dispensable. Targeting mutations in genes classically considered drivers, but at stages in which they are dispensable, will likely lead to rapid tumor editing.

\section{Sterile inflammation, immunosuppressive cells \& vasculature contribute to the tumor microenvironment}

\section{Sterile inflammation}

Inflammation is a process that also shapes tumor evolution. Anti-tumor acute inflammation is a critical process for recognition and successful clearance of cancer cells. However, the focus of this review will be contributions from chronic inflammation (also referred to as sterile inflammation), which is a complex process that contributes to the development and progression of cancer [18]. Sterile inflammation often results in the production of reactive oxygen and nitrogen species, which play a role in driving mutagenesis in cells and potentiating oncogenesis [19,20]. Similarly, some of the pro-inflammatory molecules induced during sterile inflammation will drive angiogenesis and metastases, in part by promoting the epithelial to mesenchymal transition [21-23]. This is especially critical because over $90 \%$ of cancer patient deaths are from metastases and not the primary tumor [24].

This type of sterile inflammation is, in large part, driven by senescent cells. Senescent cells represent an example of antagonistic pleiotropy: they protect the organism earlier in life, such as prevention of cancer and positive 
contributions to wound healing, but contribute to age-related pathologies and promote oncogenesis later in life [25]. While senescent cells are normally naturally cleared as they arise by inflammatory monocytes and $\mathrm{T}$ cells, organisms eventually fail to efficiently clear senescent cells as they age potentially due to an increase in the total number of these cells, a decrease in effective immune clearance, or a combination of both [26]. Cellular senescence is defined as the irreversible exit from the cell cycle, and as such these cells are not defined by a single marker, but rather by a constellation of different markers [27]. $\mathrm{p} 16^{\mathrm{INK} 4 \mathrm{a}}$ is a hallmark of senescent cells [28,29], and progressive clearance of $\mathrm{p} 16^{\mathrm{INK} 4 \mathrm{a}}$-expressing cells throughout the life of mice significantly inhibits the formation of age-related pathologies [30].

The molecular programs resulting from senescent cells is termed the senescence associated secretory program and can have pro-tumorigenic effects. This program drives a variety of pro-inflammatory, angiogenic molecules, including ones that have direct effect on cells, such as IL-6 and IL-8, as well as those that can induce senescence in neighboring cells via paracrine signaling, such as IL-1 [31-34]. Targeting senescent cells or their mediators of sterile inflammation may prove to be a more effective method of controlling further oncogenesis and metastasis and creating a more immune-permissive environment in the tumor. In a recent clinical study, CANTOS, tested an IL-1 $\beta$ targeting monoclonal antibody $(\mathrm{mAb})$, canakinumab, in patients with previous myocardial infarctions and high-sensitivity C-reactive protein levels of $>2 \mathrm{mg} / \mathrm{l}$. This study only showed modest benefits for its primary end points of reducing nonfatal myocardial infarction, nonfatal stroke or cardiovascular death [35]. Interestingly, the trial showed a reduction in cancer-associated mortalities, in particular lung cancer mortality, across different doses [35,36]. While not the primary endpoint of the study, this is suggestive that targeting some sterile inflammation mediators may have a beneficial effect in oncology, especially in combination with other therapies that induce de novo $\mathrm{T}$ cells.

\section{Immunosuppressive cell populations}

The tumor microenvironment (TME) is a highly complex, immunosuppressive structure comprised of numerous cell types and soluble factors that effectively inhibit antitumor T-cell responses. TME development is key to allowing the tumor to grow and evolve. Cell types making up the TME include, myeloid derived suppressor cells (MDSCs), regulatory $\mathrm{T}$ cells (Tregs), invariant natural killer $\mathrm{T}$ cells, tolerogenic dendritic cells (DCs), type 2 neutrophils, suppressive B-cell populations and tumor-associated macrophages. For this section we will focus on two major populations, MDSCs and Tregs.

Immature myeloid populations usually migrate to the site of stress following infection or trauma in order to replenish local DC populations. At the tumor site, soluble factors associated with inflammation and angiogenesis lead to the aberrant differentiation of these immature myeloid cells into MDSCs. GM-CSF, G-CSF, TGF- $\beta$, TNF- $\alpha$, VEGF, IL-1, IL-6 and IL-10 have all been shown to drive differentiation, survival and activation of MDSCs [3739]. In addition, the hypoxic environment with increased concentrations of lactic acid and adenosine, have been shown to prevent maturation of antigen-presenting cells (APCs) and further drive MDSC differentiation [40,41]. MDSCs are a heterogeneous population of suppressive myeloid cells and the markers differentiating each subset are complex and vary between mouse and humans [39]. To add further complexity, not all MDSC populations are immunosuppressive [42]. At a population level, MDSCs can orchestrate the formation of the immunosuppressive TME through cross-talk and recruitment of the various other immunosuppressive cell types mentioned above. Their effector function is driven by suppressive cytokines (IL-1 and IL-6), enzymes leading to suppressive metabolites (Arginase and IDO) and cell surface molecules (PD-L1/2)[38].

Tregs present in the TME include both thymically derived and induced subsets. Accumulation of both of these Treg subsets in the TME occurs through migration and de novo development (mediated by IL-10 and TGF- $\beta$ ), both of which can be driven by MDSCs. Furthermore, numerous animal tumor models show that MDSCs support the function and activation of Tregs [43,44]. Tregs mediate the suppression of anti-tumor T-cell responses by secreting immunosuppressive cytokines as well as contact-dependent suppression through cell surface molecules.

Preconditioning therapies, prior to vaccination should focus on targeting these populations. Evidence of the benefit to targeting MDSCs is shown by the use of sunitinib, a multi-targeted receptor tyrosine kinase inhibitor approved for use in renal cell carcinoma and gastrointestinal stromal cancers. Sunitinib has been shown to reduce the generation and activity of MDSCs and reduces Tregs in the TME [45,46]. Many clinical trials are currently testing the effect of targeting MDSCs in combination with other immunotherapies by targeting their development, migration and effector function [47]. Depletion strategies will vary in efficacy depending on the type of MDSC targeted; for example, gemcitabine has been shown to preferentially target the non-suppressive $\mathrm{CD}_{3}{ }^{-} \mathrm{CD}_{11 \mathrm{~b}^{+}}$ 
CD14- CD15- MDSC populations [42]. Preclinical models have shown that targeting CTLA-4 and PD-1 pathways will inhibit the effector function of both MDSCs and Tregs [48-51]. Similarly, therapeutics targeting the soluble factors that lead to the differentiation of MDSCs and Tregs as well as those involved in mediating their effector functions have shown benefit in preclinical studies [48]. Finally, Toll-like receptor (TLR) agonists (TLR 3, 7, 8 and 9) have been shown to diminish the suppressive activity of MDSCs [52]. Removal of MDSCs in particular, will be crucial in reversing the immunosuppression in the TME and establishing an environment to support the continued function of effector T cells and APCs within the tumor and draining lymph node (Figure 2).

\section{Modifying tumor vasculature}

Following successful generation of de novo anti-tumor $\mathrm{T}$ cells, specifically able to target stably-expressed tumor proteins, migration of these cells to the tumor mass is key to achieving tumor regression. Combination approaches will be necessary in preconditioning patients to allow for infiltration into the TME, as the tumor vasculature is often non-permissive to efficient migration [53]. Aberrant expression of VEGF promotes development of new vasculature to help oxygenate and deliver nutrients to a growing tumor [54]. However, this vasculature can provide a physical barrier preventing the infiltration of different types of immune cells [53] (as well as inducing T-cell exhaustion [55] and promoting the differentiation of, the previously discussed, immunosuppressive cell types in the TME). It can also hinder diffusion of therapeutic agents into the tumor environment, with larger molecules being more susceptible [53]. Anti-angiogenic therapies, often large or small molecules targeting the VEGF signaling pathway, have shown promise in normalizing the tumor vasculature [56]. To date, clinical trials involving antiangiogenic therapeutics in combination with vaccines are scarce and have reported mixed success [57-59]. Modeling combinations of anti-angiogenic drugs/mAbs and vaccines in small animals is difficult due to species-specific differences in drug/target interactions and immunogenicity of vaccine targets, which likely leads to clinical trials with suboptimal dosing and timing of the combination. As more clinical data is generated using vasculaturetargeting agents in combination with vaccines, our understanding of the timing and dosing of preconditioning regimens utilizing these therapeutics will improve. It is also possible that, in the context of a staged approach, these agents will not need to be co-administered, potentially simplifying dosing considerations.

\section{Antigen selection for use in oncology vaccines}

Ultimately, by targeting sterile inflammation, immunosuppressive cell populations and aberrant vasculature, we should be able to promote entrance and function of de novo $\mathrm{T}$ cells. In the next section, we will describe vaccine-based approaches to induce de novo $\mathrm{T}$ cells in oncology settings. Two major factors contribute to the immunogenicity and subsequent effectiveness of vaccine approaches. First, the quality of antigen encoded is critical for both inducing the desired type of CD8 or CD4 T cell as well as reducing the formation of inhibitory Tregs. Second, the antigen must be presented in an inflammatory context to drive a robust, durable and high-quality T-cell response that is more resistant to peripheral tolerance.

\section{Targeting personalized neoantigens for vaccines}

The discovery that cancer cells express, potentially immunogenic, mutated self-proteins has been known since the early $20^{\text {th }}$ century [60]. However, the technological advances in nucleic acid sequencing technologies in the last couple of decades has allowed cost-effective, large-scale sequencing of tumor cells, expanding the list of possible immunogenic neoantigen targets. This has reinvigorated interest in cancer vaccination because it is now possible to vaccinate against non-self antigens while producing no on-target autoimmune effects. This is a key benefit of this approach compared with TAAs, where on-target toxicity has been historically viewed as a hurdle for this approach. Clinical trials in melanoma and glioblastoma demonstrate proof-of-concept that anti-tumor T-cell priming is possible using neoantigen vaccines and when combined with checkpoint blockade inhibitors, complete clinical responses are achievable [61-65].

Personalized neoantigen therapies have become increasingly popular in recent years and focus on in silico prediction methods integrating genomics and proteomics. These approaches have been reviewed here [66,67]. The functional outcome of the popularity of this approach, is the field focusing on new methods of designing algorithms and improved integration of steps involved in the antigen processing pathways [68]. Alternatively, some have instead preferred more unbiased approaches [69]. Regardless of the approach, this has resulted in forced rank lists which, by definition, do not take the biology of targets into account and focuses mostly on passenger mutations. While some approaches also integrate shared neoantigens, candidates are selected based on drug developability and not 


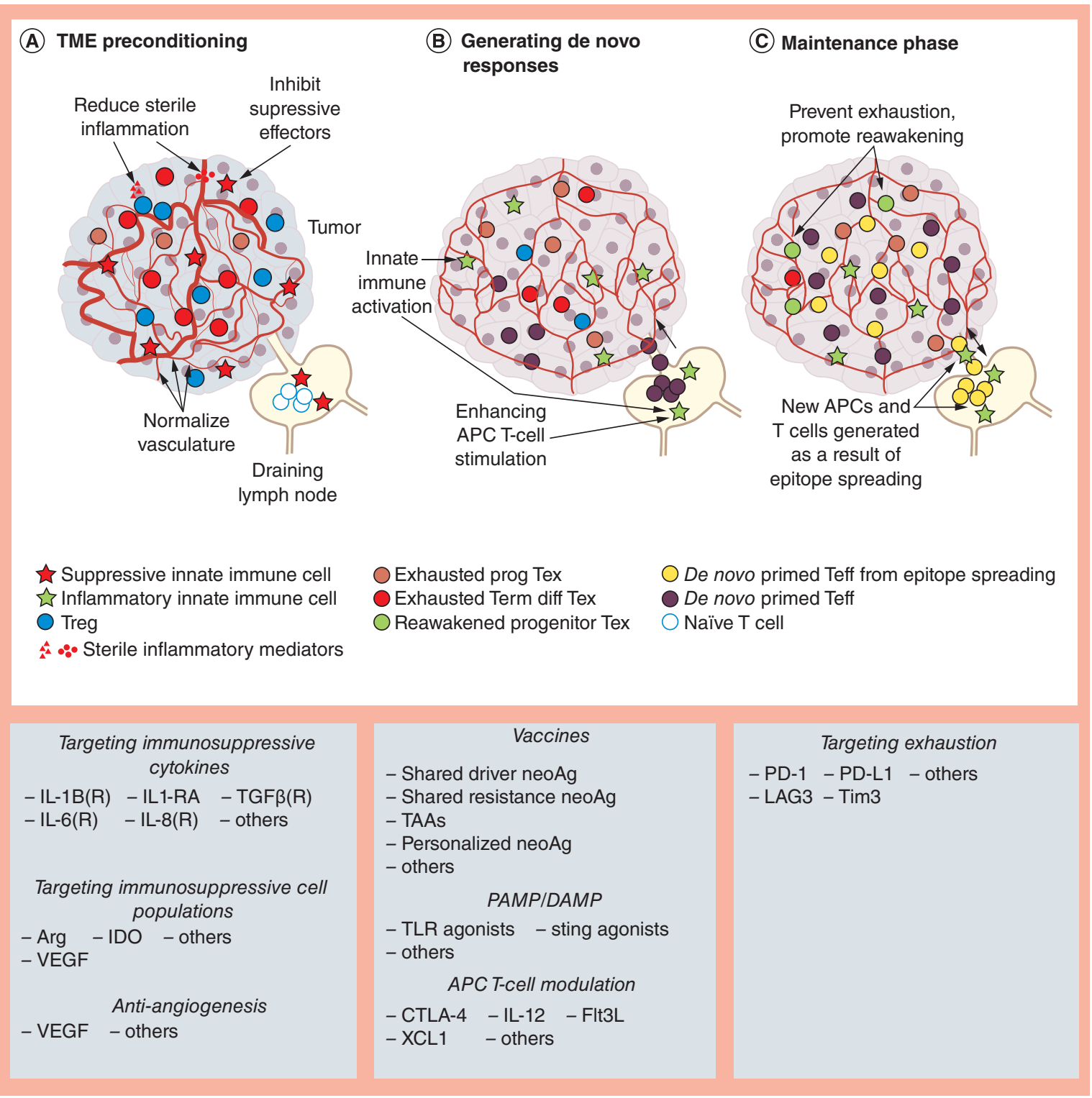

Figure 2. Preconditioning model for cancer vaccine combination therapy. (A) Prior to cancer vaccination, the suppressive tumor microenvironment is preconditioned to provide the optimal environment for priming, migration and effector function of T cells. Small molecule inhibitors, protein agonists/antagonists and monoclonal antibodies targeting suppressive cytokines and cell populations reverse the inhibitory environment. Anti-angiogenic inhibitors targeting VEGF can induce vasculature normalization, preconditioning the blood vessels for efficient migration of cells to and from the draining lymph node. In addition, anti-VEGF prevents the survival and differentiation of numerous immunosuppressive cell types in the tumor microenvironment. (B) Cancer vaccination and/or immune modulators targeting the APC T-cell interaction are combined to prime de novo T-cell responses. (C) Anti-exhaustion checkpoint inhibitors follow vaccination to re-awaken exhausted progenitor $\mathrm{T}$ cells and protect de novo primed T cells from becoming dysfunctional. Tumor killing results in epitope spreading, broadening the anti-tumor T-cell response to antigens not included in the vaccine.

APC: Antigen-presenting cell; TAA: Tumor-associated antigen; TME: Tumor microenvironment; TLR: Toll-like receptor.

biological function. As discussed above, we believe that focusing on and stratifying stably expressed proteins is a superior approach for combination therapies. 


\section{Targeting stably expressed proteins}

Both shared neoantigens and TAAs fall within the category of stably expressed proteins when controlled for a given clinical stage of a tumor. Shared neoantigens can be broadly separated into shared driver mutations and shared resistance mutations arising in response to therapies. In this first section, we will focus on potential shared neoantigen subsets as targets. We will then address how TAAs could add a complementary dimension to combination treatments.

\section{Shared driver mutations}

As monotherapies, clinical trials focusing on shared driver mutations have had mixed success and are thus difficult to interpret. The earliest trials have mainly focused on common hotspot mutations in the RAS oncogene as well as $p 53, E G F R v I I I$ and more recently IDH1 across a range of malignancies [70-74]. These clinical trials are hard to collectively assess since multiple vaccine platforms have been used across a range of different patient populations, tumor types and stages. Most of the trials have avoided preselection of MHC genes in patient populations, meaning that enrollment of patients with HLA alleles capable of binding and presenting the shared epitope is down to luck. Furthermore, combination approaches with immune modulators have only recently become common in clinical trials, which makes comparisons to historical trials difficult (see below combination therapy section). An additional consideration is the timing of administration. As discussed previously, targeting driver mutations at the wrong clinical tumor stage makes them more susceptible to immunoediting, thus behaving more as a passenger mutation.

Our goal is to identify clinical stages where shared driver mutations have stable expression to enable both therapeutic and prophylactic vaccination. Patients within that clinical stage are excellent candidates for therapeutic vaccines, whereas patients at a high risk to develop certain shared driver mutations as their cancer progresses pose a unique treatment window that is currently underexploited. To identify such mutations, we can leverage large datasets of mutations that are publicly available, such as The Cancer Genome Atlas, that are rapidly expanding [75]. We can then pair this data with empirically determined compatibility with HLAs using assays involving patient samples. An example of this approach was recently described, using The Cancer Genome Atlas and COSMIC databases, to demonstrate a proof-of-concept using patient derived peripheral blood mononuclear cells [76]. The time taken to validate shared neoantigen candidates in these patients was 20 days, in comparison to the quickest reported personalized neoantigen clinical trial which reported a median time of 103 days [63]. This framework will help integrate data as it becomes available, and to better understand kinetic analysis of the tumor mutanome allowing for further refinement of combination approaches for eligible patients.

\section{Shared resistance neoantigens}

As mentioned above, the tumor is constantly evolving due to selective pressure from the environment and immune system and as such, vaccines targeting tumor mutations suffer from a possibility of antigen loss [77]. However, tumor evolution can be exploited for vaccination purposes if it is possible to predict the neoantigen before it arises. Following the advent of targeted therapies, frequently using small molecules to target driver mutations that confer a growth advantage to the tumor, patients that initially responded to these therapies would often relapse and become resistant $[17,78]$. The tumor mutates in response to the therapeutic agent and acquires a compensatory mutation to bypass the selective pressure. Often these resistance mutations are found in the same residue among patients receiving the same therapy and, as the secondary mutation arose in response to a small molecule and not immune editing, there is a chance that this mutation may be immunogenic. The ability to predict these mutations that arise in multiple patients allows another unique opportunity to create a universal, prophylactic cancer vaccine.

Anti-estrogen therapy in breast cancer provides a good opportunity to vaccinate against a resistance mutation. $70 \%$ of breast cancer patients are treated with anti-estrogen therapies and $20 \%$ of patients with estrogen receptor positive, metastatic disease develop gain-of-function mutations in ESR1 [79]. Mutations arising in a hotspot region (commonly at residues D538 and Y537) of the ligand binding domain of ESR1 leads to constitutive activation of the receptor and resistance to anti-hormone therapy [80]. Immunization targeting multiple ESR1 mutations has shown efficacy in mouse models of breast cancer providing a basis for clinical translation [81]. ESR1 mutations, along with resistance mutations found in EGFR (following erlotinib/gefitinib treatment) as well as mutations leading to splice variants arising in the androgen receptor following anti-androgen treatment of prostate cancer, represent unique opportunities of shared neoantigens with predictable expression patterns that can be prophylactically vaccinated against. As more therapies (both vaccine and non-vaccine) targeting tumor mutations are used to treat patients, the 
list of predictable resistance mutations is likely to increase. Resistance mutations are an underappreciated source of shared neoantigens that, we predict, will form the basis of effective prophylactic vaccines in the future.

\section{Targeting TAAs}

The use of TAAs as vaccine targets is beginning to be phased out in favor of more immunogenic neoantigens based on the reasons described above. However, despite the limitations of TAAs as a sole target to build a vaccine around, a carefully selected group will have a use in cancer vaccines. A number of TAAs have shown promise in clinical trials by generating immune responses, if not resulting in complete clinical responses. As long as the TAA does not initiate an autoimmune response, there is no disadvantage for their inclusion in cancer vaccines targeting shared neoantigens.

Breast cancer is a disease that serves to exemplify the challenges facing TAA-based vaccines. HER 2 is overexpressed in around $20-30 \%$ of breast cancer cases and clinical success has been achieved targeting this molecule with a number of immunotherapies, most notably the mAbs trastuzumab and pertuzumab. Preclinical vaccine data targeting HER2 reveals strong CD8 T-cell responses leading to the majority of vaccines being based around class I epitopes [82]. Peptide-based vaccines targeting a 9 amino acid long (9mer) MHC class I epitope (HLA-A2/A3 restricted), called E75, have been extensively studied in the clinic in addition to another short 9mer peptide vaccine, GP2 [83-85]. Short peptide, minimal epitope vaccines have struggled to elicit efficacious anti-tumor T-cell responses due to poor cross presentation, external loading on MHC of nonprofessional APCs and no induction of CD4 T-cell responses as opposed to synthetic long peptides [86]. Clinical data suggests that anti-HER2 CD4 T cells correlate with complete responses and as such, should be added to vaccine designs [87]. In addition to peptide-based vaccines, numerous other platforms have been used or are currently in clinical trials including DCs, DNA and viral vector-based platforms. Some of the viral vector-based platforms, reviewed in further detail below, have had more promising early stage clinical results [88]. Overall, most anti-HER2 vaccines have shown safety in early clinical trials and struggled to show efficacy in later stages. Frequently, studies have focused on metastatic patients, similar to early anti-HER $2 \mathrm{mAbs}$ trials, whereas the most positive clinical efficacy results have come from patients with resected disease [89]. HER2 is a good example of a TAA vaccine showing limited efficacy in clinical trials due to poor immunogen design and vaccine platforms. The presence of immunogenic CD8 epitopes, restricted to the common HLA-A2 allele, makes HER2 a valuable tool in cancer vaccine strategies. Combination of these epitopes, along with shared neoantigens such as, the previously discussed, ESR1 mutations, into newer platforms will likely produce an immunogenic vaccine. Combined with the large literature of HER2 vaccine clinical trials, a new vaccine targeted to certain patient populations will have a high chance of clinical success.

$p 16^{I N K 4 a}$ is a tumor suppressor gene expressed in a variety of cancers including $90 \%$ of cervical cancers and $50 \%$ of head and neck cancers [90]. Mutations in $p 16^{I N K 4 a}$, as with many loss-of-function mutations, can be varied. $p 16^{I N K 4 a}$ is also a hallmark of senescent cells, and removal of $p 16^{I N K 4 a}$-expressing cells is sufficient to prevent senescent-related phenotypes from arising [30]. Targeting $p 1 \sigma^{I N K 4 a}$-expressing cells could help prevent tumors from metastasizing and reduce sterile inflammation creating a more immune-permissive environment around tumors. Recent Phase I/IIa vaccine trials both as a monotherapy and in combination with cisplatin-based chemotherapy with $p 16^{I N K 4 a}$ peptides adjuvanted with ISA51 showed safety and tolerability, with most patients showing T-cell responses (mostly CD4) and antibody responses, despite the lack of HLA matching in the study (clinical trials NCT02526316 and NCT01462838). While these studies have yet to be progressed, this demonstrates the feasibility of including proteins that allow immune targeting of senescent cells in future cancer vaccines.

$\operatorname{Trp} 53$, or $p 53$, is a master transcriptional regulator that functions as a tumor suppressor in normal cells and is mutated in around 50\% of cancers [91]. Mutations in $p 53$ are numerous and lead to loss-of-function which promotes cell cycle dysfunction and tumorigenesis [92]. Mutations lead to elevated expression of p 53 protein levels thus establishing it as both a potential TAA and neoantigen target. Unfortunately, many of the mutations occurring in $p 53$ often reduce the affinity to bind to common HLA class I alleles in comparison to the wild-type version [93]. This fact, combined with the observation of endogenous T-cell responses in many cancer patients against wild type $p 53$, have led to many vaccines targeting $p 53$ as a TAA rather than a neoantigen [94]. Numerous clinical trials have been reported using a variety of vaccine platforms (synthetic long peptides, DCs and viral vectors) and report a familiar story of induction of immune responses with weak clinical outcomes unless combined with immune modulating agents [95-98].

Despite lack of clinical success so far, $\mathrm{p} 53$ is an attractive antigen to include in a vaccine due to its function as a driver gene. Similar to $\mathrm{p} 16^{\mathrm{INK} 4 \mathrm{a}}$, mutant $\mathrm{p} 53$ can influence the extracellular environment through metabolic 
remodeling, promoting inflammation and leading to the creation of the immunosuppressive TME [99,100]. The inclusion of $\mathrm{p} 53$ in a polytopic vaccine can serve as an additional antigen target that has the benefit of being stably expressed at a high level and actively modeling the immunosuppressive TME.

Taken together, we can now start to assemble a picture on the rational design of treatments for certain subpopulations of patients. By way of example, breast cancer patients could have a vaccine with containing the following components. First, shared driver neoantigens such as mutations in PIK3CA could be included, which is commonly mutated in breast cancers and contributes to proliferation [101]. Next, HER2, which encourages the growth of cancerous cells could be included as a TAA if patients are HER2 ${ }^{+}$or are at risk to overexpress HER2 [88]. If patients are candidates for aromatase inhibitors, the vaccine could include ESR1 mutations [81]. Targeting ESR1, which confers resistance to aromatase inhibitors, could help extend their window of therapeutic effectiveness. Finally, p16 ${ }^{\mathrm{INK} 4 \mathrm{a}}$ and $\mathrm{p} 53$ components to the vaccine could help reduce the likelihood of metastases. This would represent a vaccine that allows for nontoxic targeting of multiple pathways for inclusion in a combination therapy and helps reduce the potential for tumor escape.

\section{Technologies to target antigens}

Platforms impact the context in which the antigen is presented to the immune system through biodistribution and cell types expressing the antigen, local tissue microenvironments at the site of delivery, and cell-extrinsic and -intrinsic impacts on host cells expressing the proteins. There are a few major classes of modalities that are used for the purposes of cancer vaccines: cell-based, non-replicating technologies (DNA, conventional non-replicating mRNA, peptides) and virally derived vectors (single cycle particles, replication-competent particles and synthetic self-replicating RNAs). Cell-based methods for vaccination have been reviewed elsewhere [67], and for the purposes of this review our focus will be on the use of non-replicating and virally derived vaccines in oncology.

\section{Biodistribution \& interaction with host cells}

Biodistribution is primarily affected by the drug product itself, as well as the method and route of administration. For virally derived platforms, differential usage of host-cell receptors creates a natural tropism for vectors such as human adenoviruses, which impact biodistribution of the vaccine [102-105]. For clinical RNA-based technologies, biophysical characteristics such as nitrogen-phosphate $(\mathrm{N}: \mathrm{P})$ ratio during formulation and PEG or cholesterol compositions can impact biodistribution, potentially encouraging differential levels of circulation [106-108]. For peptide- and nucleic acid-based technologies, formulations may have an impact on local depot effects, both inside and outside of cells affecting kinetics of cargo release. Furthermore, route of injection can impact the types of cells that can be directly transfected or transduced with the vaccine gene delivery vector. Intradermal injection routes are thought to heighten the probability of directly transfecting or transducing APCs, and skin-resident DCs may be superior at driving certain responses $[109,110]$. Intramuscular injection would primarily involve delivering genes to myocytes, with tissue resident macrophages and DCs playing a prominent role as APCs [111,112]. Similarly, subcutaneous injections would primarily deliver material to fibroblasts. In all of these cases, some percentage of the material likely makes its way directly to draining lymph nodes [113]. In contrast, intravenous injections are thought to deliver a large amount of the material to the lungs, spleen or liver, depending on the characteristics of the drug product [108]. However, studies tend to compare injection routes for the same vaccine modality. More studies would be required making direct comparisons between platforms using the same injection route to disentangle whether differences in immunogenicity are based on injection route or specific to modalities. For instance, this would help us to understand whether intradermal administration encourages superior immunogenicity for all vectors due to the composition of immune cells at the site of injection, or just enhance conventional non-replicating RNAs due to their lower ceiling of immunogenicity.

Once inside the host cell, there may be both cell-extrinsic and -intrinsic platform-specific effects impacting development of subsequent immunity. Differential abilities to activate host intracellular viral sensing mechanisms such as RIG-I, MDA5, TLRs, NODs, PKR, RNase L or 2'5'-OAS is directly dependent on the composition of the vector. Drug substance manufacturing steps, such as removing double-stranded RNA from conventional nonreplicating mRNAs, would be expected to result in poor induction and recognition by many of these pathways [114]. In contrast, self-replicating RNAs, which go through a double-stranded intermediate as part of the genomic amplification process, may strongly activate these pathways $[115,116]$. Viral platforms often encode host cell innate immune evasion mechanisms in both their structural and nonstructural regions [117]. How these evasion mechanisms impact immunity in vaccine vectors is often poorly characterized and the subsequent effect on immunity in specific 
contexts (e.g., cancer versus acute viral infections) is rarely assessed. Collectively, these mechanisms are critical when designing combinations. Whereas combinations including immune modulators, such as TLR agonists, that induce type I interferons (IFNs) may be beneficial for RNA-based vaccines, excessive stimulation of IFN pathways may inhibit the response to both RNA-based vaccines and virally derived vectors [118-120]. Similarly, peptides have poor induction of innate immunity and usually rely on the presence of co-administered or conjugated adjuvants to bolster their immunogenicity [121].

Cell-extrinsic effects from host-cell activation are driven by the induction of pro-inflammatory chemokines or cytokines induced directly by the technology, through the formulation, or a combination of both. Chemoattractants can alter how many APCs migrate to the local site of injection to pick up antigen [122,123]. Furthermore, some chemokines and cytokines can affect the maturation of APCs and affect their molecular imprinting, which may bias downstream immune cell differentiation decisions [124]. Differential activation of host cells can also affect upregulation of costimulatory molecules, which further alters the quality of activation of APCs [125]. Virallyderived platforms have been shown to have strong induction of pro-inflammatory molecules [115,116], whereas nonreplicating modalities have typically relied on adjuvants or injection devices to help induce those pathways [121,126]. Beyond cell-extrinsic effects, differences in cell-intrinsic effects should also be taken into consideration for different vaccine gene delivery platforms. Clonal expression of antigens can vary depending on the technology. Whereas antigen expression from conventional non-replicating mRNAs is proportional to the amount of copies directly delivered to a cell, virally derived RNAs that self-replicate (replicons) will have a higher level of protein expression and longer expression kinetics in each cell where it productively launches [127]. Additionally, activation of host cell innate immune activation can result in shutdown of host cellular translation via PKR or encourage degradation of mRNA through RNase L activation [115,128]. Host-cell activation can also alter proteasomal processing, which may result in different epitopes being presented and is a potential source of bias for polytope approaches being currently utilized for neoantigen vaccines [129,130]. Currently, it is unknown to what extent design of the gene cassette for polytopes will impact processing of individual epitopes.

\section{Shaping of adaptive immune responses}

Biodistribution, microenvironment at site of infection and alteration of the host cell all shape the downstream adaptive immune responses. In oncology, an increasingly used virally derived technology are single cycle, replicationdeficient adenoviruses derived from a variety of species, including human, chimpanzees and great apes (clinical trial NCT04041310) [131-133]. A common feature of these platforms is the capacity to induce a high quality and high magnitude of T-cell responses [131]. However, these vectors may also induce anti-vector immunity, which decreases the potency and feasibility of repeat dosing, a common feature of oncology vaccine regimens [134]. To circumvent this issue, there is an increased use of additional virally derived or non-replicating technologies in heterologous prime-boosts approaches, which may also bolster the quality and magnitude of both effector and memory responses. Both modified vaccinia ankara and synthetic alphavirus-based replicons encoding neoantigens are currently being evaluated in Phase I/IIa clinical trials in a personalized approach (clinical trials NCT04041310 and NCT03639714). This heterologous prime-boost vaccine regimen relies on a chimp adenovirus prime followed by multiple synthetic replicon-based boosts, or a great ape adenovirus prime followed by multiple modified vaccinia ankara boosts (clinical trials NCT04041310 and NCT03639714). There has been evidence in the infectious disease field that heterologous prime-boosts approaches yield increased T-cell responses in humans for Ebola and Malaria [135,136], but whether this translates to humans in oncology settings is still under investigation. Despite the improvement to immunological responses, a major drawback of heterologous prime-boost approaches is the high cost of goods and complexity of manufacturing to generate two completely different drug products.

Self-replicating RNAs have also been utilized in homologous prime boosts approaches in oncology. Viral replicon particle-based vaccines have had promising results in human Phase I/IIa clinical trials targeting CEA, PSMA and HER2 generating both antibody and T-cell responses $[88,137]$. In addition to these clinical targets, the platform has also shown efficacy across a wide range of TAAs in preclinical small animal models [138]. Both clinical and preclinical studies have shown the ability for this type of platform to induce a high magnitude and quality of both MHC class I and class II responses, as well as generating high titers of antibodies [138]. Nevertheless, to date, no homologous prime-boost approach for either infectious disease or oncology has been used clinically for fully synthetic self-replicating RNAs. However, the low cost of goods, relatively simple manufacturing and the ability to encode multiple molecules useful in multidimensional therapies, make synthetic replicons an ideal platform for use in oncology. 
The most commonly used platforms in oncology have been non-replicating technologies including DNA, peptides and conventional mRNA. Historically, DNA vaccines were chosen due to their ability to elicit robust CD8 ${ }^{+}$ T-cell responses. However, DNA vectors suffer from difficulty of delivery and currently rely on electroporation technologies, which have their own challenges for achieving widespread consistency [139]. Additional considerations include fragility of devices, requirement for additional training to use and operator variability in the field. Additional concerns exist regarding whether DNA vaccines are able to induce sufficient levels of $\mathrm{CD}^{+}{ }^{+} \mathrm{T}$ cells or antibody responses. Comparatively, DNA vaccines currently have a high cost of goods due to the poor scalability between small animals and humans often requiring multigram doses clinically to see an effect [140].

RNA-based medicines previously suffered from a lack of stability and a predisposition for degradation limiting their clinical development [141]. These issues have largely been solved and non-replicating mRNAs have been in a variety of Phase I/IIa and Phase II trials for both neoantigens and TAAs. The Phase II clinical trials from Curevac testing the efficacy of CV9104 in prostate cancer were discontinued after failing to hit their primary endpoints (clinical trial NCT01817738), which could be partially attributed to a nonoptimized formulation. Clinical trials in neoantigens have multiplied in recent years and seem to rely on regimens that involve injections with short intervals (e.g., 1 week in some cases). These intervals would likely inhibit production of memory $\mathrm{T}$ cells and longer-term immunity [142] and are likely being chosen due to the poor immunogenicity of the platform. Reactogenicity and other adverse events are often seen at doses starting at 100 micrograms, largely due to the lipid particles, which may make higher doses more challenging from a safety perspective [143,144].

Peptides are the most heavily utilized non-replicating technology in oncology. Historically, attempts to generate peptide-based vaccines in infectious diseases have not resulted in any products. This may be because peptides on their own are weakly immunogenic and often fail to elicit sufficiently high antibody or T-cell responses to confer protection [145,146]. Current approaches focus on adjuvants and DC delivery as ways to circumvent this issue, but have still been met with limited success $[145,146]$. In fact, there are over 450 clinical trials utilizing peptides, with no approved commercial products to date [146]. The vast majority of these trials are in Phase I and Phase II for a variety of indications. Nevertheless, adjuvanted peptides have an excellent safety profile in humans and creating cGMP peptides is a straightforward and rapid path to generate proof-of-concept data in humans [146]. While generating peptides for a large population is feasible, generating peptides for personalized approaches may yet be challenging due to the costs and timing caused by current technological limitations for peptide synthesis [61]. Another complication is potential biasing of T-cell responses. In a recent unpublished Phase Ib clinical trial, NEOPV-01 (synthetic long peptides adjuvanted in Hiltonol) combined with anti-PD-1 generated detectable CD4 responses from $42 \%$ of epitopes versus CD8 responses from 28\% of epitopes in 11 patients. This confirms the Class II bias that has been previously thought to occur. This is especially notable since most prediction algorithms for personalized neoantigens are much more successful at predicting class I compared with class II epitopes, despite efforts to improve the algorithms [147]. While being a strong technology for obtaining proof-of-concept data about the immunogenicity of different antigens in humans, this technology as currently designed is less likely to be successful compared with virally derived approaches.

\section{Treatment staging \& designing the next wave of multidimensional therapies}

Successful staging of agents in a combination regimen will be critical for achieving desired outcomes. Agents that precondition patients, induce de novo responses or sustain existing responses have had limited success as monotherapies. The major focus to date has been on immunotherapies that sustain existing responses (such as anti-PD1 therapy) or induce de novo responses (anti-CTLA-4 therapy and vaccine-based approaches). However, we believe this is backward. Without a sufficient focus on first preconditioning patients, these approaches are less likely to succeed. An instructive example of staging comes from chronic hepatitis B infection. Recent clinical trials from Replicor (Montreal, Quebec) have shown that nucleic acid polymers reducing immunosuppressive HBsAg, followed by PEG-IFN treatment promotes seroconversion and ALT flares, which are indicative of T-cell responses. Treatment with PEG-IFN or nucleic acid polymers alone do not have this effect, showing the importance of combinations that reduce an immunosuppressive microenvironment prior to immune-inducing therapies. While T-cell dysfunction and exhaustion within the TME may arise from a different set of factors, this nevertheless provides an encouraging demonstration of the concept. To this end, we will outline examples of how preconditioning, de novo immune induction and maintenance of existing responses can be combined (Figure 2). 


\section{Preconditioning}

For successful vaccination in established tumors, we must first counter the immunosuppressive TME to prevent dysfunction and exhaustion of new immune cell entrants (Figure 2). The extent to which this immunosuppression can be reversed, and the number and type of therapies used, will depend on disease stage. These combinations could focus on remodeling vasculature to promote entrance to the tumor, disrupting paracrine signaling networks driving sterile inflammation, and direct therapeutic targeting of immunosuppressive cells. As mentioned previously, both anti-VEGF and anti-IL-1 antibodies have shown encouraging results in restoring vasculature, inhibiting immunosuppressive cells and potentially reducing metastases deriving from sterile inflammation respectively. Recently, VEGF was reported to drive T-cell exhaustion in colorectal cancer and blocking this pathway restored the anti-tumor function of T cells [55]. Additionally, IL-1 receptor antagonist is being used in rheumatoid arthritis [148] and anti-IL-6 [149] has been approved, while anti-IL-8 (clinical trial NCT02536469) antibodies are in clinical trials. Each of these make for attractive candidates to test in preconditioning regimens. Furthermore, recent clinical trials are focusing on targeting the development, migration and effector function of immunosuppressive cells in the TME [48]. Targeting many of the soluble factors that promote sterile inflammation, angiogenesis, Treg differentiation and cell surface inhibitory molecules has shown promise in preclinical models and in patients. Similar to the role of preconditioning chemotherapy regimens prior to adoptive cell therapies, reversing the TME suppressive environment will create a more permissive niche for the expansion of newly primed anti-tumor $\mathrm{T}$ cells, which could then be supported later with another round of anti-exhaustion checkpoint inhibitors (CPIs). Finally, dosing of many of these agents will need to be determined experimentally in patients with disease as many of these therapies have differential effects depending on dose. For example, low doses of cyclophosphamide will preferentially deplete Tregs rather than effector T cells due to differential intracellular levels of ATP [150].

\section{Generation of de novo responses or reawakening existing responses}

The goal of therapeutic tumor vaccination is to generate anti-tumor T-cell responses which can be achieved in two ways; de novo priming of naive $\mathrm{T}$ cells or reawakening of existing anti-tumor $\mathrm{T}$ cells. With this in mind, and by tailoring combinations specifically to each vaccine platform, we can narrow the list of combinations to those most likely to achieve synergy. Priming de novo T-cell responses against the tumor is important for multiple reasons. First, the evolution of the tumor mutanome over time means that antigens that primed the initial immune response may be unimportant for tumor rejection following immune editing and therefore, vaccine intervention will redirect the T-cell response to more relevant antigens. Second, recent evidence suggests that very few $\mathrm{T}$ cells in the TME express a tumor-reactive T-cell receptor and therefore priming a new wave of naive $\mathrm{T}$ cells to attack the tumor is an important goal of vaccination [151]. To this end, targeting early checkpoints, such as CTLA-4, will be important. CTLA- 4 antagonists have been shown to preferentially induce expansion of naive T cells and broaden the T-cell receptor repertoire of cancer patients in comparison to PD-1 antagonists [152-154].

To achieve optimal priming, a vaccine will be most successful when combined with an immune modulating agent that affects the interaction between the T cell and APC, specifically DCs (Figure 2). As our knowledge of DC biology grows, we can exploit our understanding of the DC subsets that most efficiently cross-present antigen to CD8 $\mathrm{T}$ cells to enhance vaccination efficacy. Combinations of vaccines with immune modulating agents that induce differentiation or increase homing of cross-presenting DCs to the site of the immunogen will be crucial. BATF3-dependent type I conventional DCs (cDC1) have been shown to be an efficient cross-presenting population $[155,156]$. Transgenic expression of the chemokine XCL1 and Flt3 ligand in preclinical tumor models has shown recruitment of $\mathrm{cDC} 1$ and increased tumor efficacy through CD8 cross-priming [157]. Flt3 ligand is currently undergoing clinical evaluation in a number of trials combined with different vaccine modalities (clinical trials NCT02129075 and NCT00019396). When using PAMP/DAMP agonists, such as TLR ligands, to induce maturation of DCs as a combinatorial approach with vaccination, it is important to consider that CDC1 in humans mainly express TLR3 [158]. Similarly, it is important to consider the mechanism of action (MOA) of the vaccine platform when combining APC modulating agents to minimize redundancy. Viral-based vaccine platforms will trigger TLR and nucleic acid sensing pathways, so combination of these with TLR agonists or nucleic acid sensing agonists (e.g., STING) is likely to be redundant at best and detrimental at worst. Overproduction of type I IFN in response to the viral vaccine plus PAMP/DAMP agonist can lead to exhaustion of T cells [159,160]. Overall, considering the synergy between the biology of APC subsets and the vaccine platform MOA will yield the most effective combinations (Figure 2). 


\section{Maintaining or reawakening responses by preventing dysfunction \& exhaustion}

Finally, once we have preconditioned the tumor and de novo generated or reawakened $\mathrm{T}$ cells, we must then shield them from subsequent dysfunction or exhaustion (Figure 2). Following successful priming and migration into the TME, the second major area for combination therapies is to reawaken effector T-cell responses within the tumor mass. Typically, a PD-1 pathway blockade has been used to reverse exhaustion of $\mathrm{T}$ cells in late stage disease and this is an attractive target to combine with cancer vaccines in both early and late stage settings. Clinical data are limited using PD-1 blockade and vaccines, but early signs are encouraging. Synergy has been shown in studies vaccinating against TAA, shared driver mutations and HPV antigens with a range of vaccine platforms [161-164]. A recent study highlights an important consideration when combining CPIs with vaccines; administering anti-PD-1 blockade during priming has a detrimental effect and induces T-cell exhaustion [165]. Each immune modulating agent and each vaccine platform will exhibit unique kinetics and a deep understanding of the MOA of each of these will be required to design optimal combinatorial therapies.

As more research is conducted into the T-cell biology explaining the anti-tumor response to anti-PD-1 therapy (and all CPI in general), it reveals other pathways that may be exploited in combinatorial vaccine regimens. As an example, a series of literature has been published recently describing the expansion of PD- $1^{+} \mathrm{TCF} 1^{+} \mathrm{CXCR} 5^{+}$ CD8 T cells in response to PD-1 blockade [166-168]. A recent study has expanded on this finding to show that the PD- $1^{+}$TCF $1^{+}$population that responds can be expanded using IL-27 [169]. In doing so, it is possible to expand the CD8 memory pool that has stem-like properties. Vaccination in combination with anti-PD-1 may benefit from IL-27 addition in the future to reawaken the exhausted CD8 population. This timing aligns with the therapeutic window for anti-PD-1 therapies [165].

Preventing T-cell exhaustion is not limited to anti-PD-1 therapy. Antibodies against common exhaustion markers Tim-3, Lag-3, TIGIT and VISTA have shown preclinical success in tumor models [170-172] and are all in clinical development [173]. Each pathway is differentially expressed on tumor types and alters T-cell biology in a distinct manner or timing, as a result the new generation of CPIs will have distinct MOAs that synergize differently with vaccine platforms and tumor types/stages (Figure 2).

\section{Future perspective}

Success of multidimensional therapies will require careful selection of tumor clinical stage, antigen selection and platforms which maximize their effect, and both treatment dose and staging. These challenges in optimizing successful combinations are not trivial and will differ depending on the type of cancer and target population. Initial target populations are likely to be smaller than those targeted by catch-all personalized neoantigen approaches but are likelier to succeed. However, modeling these approaches in animals is unlikely to fully determine the exact regimen required for success in humans due to differences in activity of both the vaccine and the immune modulator in non-human species. Clinical trials will provide the best experimental environment to optimize each combination. As such, specific combinations that are likely to synergize should be first determined by the MOA of each individual therapy to limit the size and number of such trials. Nevertheless, with an increasing number of therapeutics becoming approved every year, there is an encouraging path for creating more effective multidimensional therapies.

\section{Executive summary}

In this review, we present the following insights on how to create multidimensional immunotherapies in oncology through improved integration of tumor biology and vaccine design:

- We can improve our immunotherapy approaches by rationally targeting tumor clinical stages that incorporate underlying stage-specific tumor biology.

- Patients may require preconditioning prior to immunotherapy to account for the impact of sterile inflammation, immunosuppressive cells and vasculature on the tumor microenvironment.

- For combinations incorporating vaccines, antigen selection permitting both off-the-shelf therapeutic and prophylactic immunizations would be ideal.

- Cancer vaccine antigens must be encoded in the right platform to elicit the optimal magnitude, quality, balance and durability of immune responses.

- Treatment staging, or the sequence in which combination agents are administered, needs to be rationally driven by the biology.

- Future perspectives on how we must practically approach the next generation of combination therapies. 
Financial \& competing interests disclosure

The authors are employees of Janssen Research \& Development, LCC. The authors have no other relevant affiliations or financial involvement with any organization or entity with a financial interest in or financial conflict with the subject matter or materials discussed in the manuscript apart from those disclosed.

No writing assistance was utilized in the production of this manuscript.

\section{Open access}

This work is licensed under the Attribution-NonCommercial-NoDerivatives 4.0 Unported License. To view a copy of this license, visit http://creativecommons.org/licenses/by-nc-nd/4.0/

\section{References}

Papers of special note have been highlighted as: $\bullet$ of interest; $\bullet \bullet$ of considerable interest

1. Bardelli A, Cahill DP, Lederer G et al. Carcinogen-specific induction of genetic instability. Proc. Natl Acad. Sci. USA 98(10), 5770-5775 (2001).

2. Greaves M, Maley CC. Clonal evolution in cancer. Nature 481(7381), 306-313 (2012).

3. Cahill DP, Kinzler KW, Vogelstein B, Lengauer C. Genetic instability and darwinian selection in tumours. Trends Cell Biol. 9(12), M57-M60 (1999).

4. Baeissa H, Benstead-Hume G, Richardson CJ, Pearl FMG. Identification and analysis of mutational hotspots in oncogenes and tumour suppressors. Oncotarget 8(13), 21290-21304 (2017).

5. Baugh EH, Ke H, Levine AJ, Bonneau RA, Chan CS. Why are there hotspot mutations in the TP53 gene in human cancers? Cell Death Differ. 25(1), 154-160 (2018).

6. Dunn GP, Old LJ, Schreiber RD. The three Es of cancer immunoediting. Annu. Rev. Immunol. 22, 329-360 (2004).

7. Smith MP, Rowling EJ, Miskolczi Z et al. Targeting endothelin receptor signalling overcomes heterogeneity driven therapy failure. EMBO Mol. Med. 9(8), 1011-1029 (2017).

8. Burnet M. Cancer: a biological approach. III. Viruses associated with neoplastic conditions. IV. Practical applications. Br. Med. J. 1(5023), 841-847 (1957).

9. Burnet FM. The concept of immunological surveillance. Prog. Exp. Tumor Res. 13, 1-27 (1970).

10. Burnet M. Immunological factors in the process of carcinogenesis. Br. Med. Bull. 20, 154-158 (1964).

11. Shankaran V, Ikeda H, Bruce AT et al. IFNgamma and lymphocytes prevent primary tumour development and shape tumour immunogenicity. Nature 410(6832), 1107-1111 (2001).

12. Yu HA, Arcila ME, Rekhtman N et al. Analysis of tumor specimens at the time of acquired resistance to EGFR-TKI therapy in 155 patients with EGFR-mutant lung cancers. Clin. Cancer Res. Off. J. Am. Assoc. Cancer Res. 19(8), 2240-2247 (2013).

13. Gjertsen MK, Bakka A, Breivik J et al. Ex vivo ras peptide vaccination in patients with advanced pancreatic cancer: results of a Phase I/II study. Int. J. Cancer 65(4), 450-453 (1996).

14. Umar A, Dunn BK, Greenwald P. Future directions in cancer prevention. Nat. Rev. Cancer 12(12), 835-848 (2012).

15. Li X. Dynamic changes of driver genes' mutations across clinical stages in nine cancer types. Cancer Med. 5(7), 1556-1565 (2016).

-• Assesses several cancer types and helps establish the concept of that driver genes are only expressed at certain clinical stages.

16. Gomez K, Miura S, Huuki LA, Spell BS, Townsend JP, Kumar S. Somatic evolutionary timings of driver mutations. BMC Cancer 18(1), 85 (2018)

-• Helps establish different behaviors of driver genes in aggregate showing dynamics between early arising and late arising drivers.

17. Pao W, Wang TY, Riely GJ et al. KRAS mutations and primary resistance of lung adenocarcinomas to gefitinib or erlotinib. PLoS Med. 2(1), e17 (2005).

18. Shalapour S, Karin M. Immunity, inflammation, and cancer: an eternal fight between good and evil. J. Clin. Invest. 125(9), 3347-3355 (2015).

19. Szatrowski TP, Nathan CF. Production of large amounts of hydrogen peroxide by human tumor cells. Cancer Res. 51(3), 794-798 (1991).

20. Ray G, Batra S, Shukla NK et al. Lipid peroxidation, free radical production and antioxidant status in breast cancer. Breast Cancer Res. Treat. 59(2), 163-170 (2000).

21. Colomiere M, Ward AC, Riley C et al. Cross talk of signals between EGFR and IL-6R through JAK2/STAT3 mediate epithelial-mesenchymal transition in ovarian carcinomas. Br. J. Cancer. 100(1), 134-144 (2009).

22. Bates RC, DeLeo MJ, Mercurio AM. The epithelial-mesenchymal transition of colon carcinoma involves expression of IL-8 and CXCR-1-mediated chemotaxis. Exp. Cell Res. 299(2), 315-324 (2004). 
23. Tato-Costa J, Casimiro S, Pacheco T et al. Therapy-induced cellular senescence induces epithelial-to-mesenchymal transition and increases invasiveness in rectal cancer. Clin. Colorectal Cancer. 15(2), 170-178.e3 (2016).

24. Chaffer CL, Weinberg RA. A perspective on cancer cell metastasis. Science 331(6024), 1559-1564 (2011).

25. Giaimo S, d'Adda di Fagagna F. Is cellular senescence an example of antagonistic pleiotropy? Aging Cell. 11(3), 378-383 (2012).

26. Childs BG, Gluscevic M, Baker DJ et al. Senescent cells: an emerging target for diseases of ageing. Nat. Rev. Drug Discov. 16(10), 718-735 (2017).

27. Campisi J. Aging, cellular senescence, and cancer. Annu. Rev. Physiol. 75, 685-705 (2013).

28. Ohtani N, Yamakoshi K, Takahashi A, Hara E. The $\mathrm{p} 16^{\mathrm{INK} 4 \mathrm{a}}-\mathrm{RB}$ pathway: molecular link between cellular senescence and tumor suppression. J. Med. Investig. JMI. 51(3-4), 146-153 (2004).

29. Collins CJ, Sedivy JM. Involvement of the INK4a/Arf gene locus in senescence. Aging Cell. 2(3), 145-150 (2003).

30. Baker DJ, Wijshake T, Tchkonia T et al. Clearance of $\mathrm{p} 16^{\text {Ink4a }}$-positive senescent cells delays ageing-associated disorders. Nature 479(7372), 232-236 (2011).

31. Acosta JC, Banito A, Wuestefeld T et al. A complex secretory program orchestrated by the inflammasome controls paracrine senescence. Nat. Cell Biol. 15(8), 978-990 (2013).

32. Acosta JC, O’Loghlen A, Banito A et al. Chemokine signaling via the CXCR2 receptor reinforces senescence. Cell 133(6), 1006-1018 (2008).

33. Coppé J-P, Patil CK, Rodier F et al. Senescence-associated secretory phenotypes reveal cell-nonautonomous functions of oncogenic RAS and the p53 tumor suppressor. PLoS Biol. 6(12), 2853-2868 (2008).

34. Coppé J-P, Desprez P-Y, Krtolica A, Campisi J. The senescence-associated secretory phenotype: the dark side of tumor suppression. Annu. Rev. Pathol. 5, 99-118 (2010).

35. Ridker PM, Everett BM, Thuren T et al. Antiinflammatory therapy with canakinumab for atherosclerotic disease. N. Engl. J. Med. 377(12), 1119-1131 (2017).

36. Gottschlich A, Endres S, Kobold S. Can we use interleukin-1 $\beta$ blockade for lung cancer treatment? Transl. Lung Cancer Res. 7(Suppl. 2), S160-S164 (2018).

- References how treatment of patients with the anti-IL-1 $\beta$ monoclonal antibody (mAb) Canakinumab reduced cancer-induced mortalities, especially for lung cancers.

37. Meyer C, Sevko A, Ramacher M et al. Chronic inflammation promotes myeloid-derived suppressor cell activation blocking antitumor immunity in transgenic mouse melanoma model. Proc. Natl Acad. Sci. USA 108(41), 17111-17116 (2011).

38. Gabrilovich DI, Nagaraj S. Myeloid-derived suppressor cells as regulators of the immune system. Nat. Rev. Immunol. 9(3), 162-174 (2009).

39. Umansky V, Sevko A. Tumor microenvironment and myeloid-derived suppressor cells. Cancer Microenviron. Off. J. Int. Cancer Microenviron. Soc. 6(2), 169-177 (2013).

40. Gabrilovich DI, Ostrand-Rosenberg S, Bronte V. Coordinated regulation of myeloid cells by tumours. Nat. Rev. Immunol. 12(4), 253-268 (2012).

41. Corzo CA, Condamine T, Lu L et al. HIF-1 $\alpha$ regulates function and differentiation of myeloid-derived suppressor cells in the tumor microenvironment. J. Exp. Med. 207(11), 2439-2453 (2010).

42. Santegoets SJAM, de Groot AF, Dijkgraaf EM et al. The blood mMDSC to DC ratio is a sensitive and easy to assess independent predictive factor for epithelial ovarian cancer survival. Oncoimmunology. 7(8), e1465166 (2018).

43. Yang R, Cai Z, Zhang Y, Yutzy WH, Roby KF, Roden RBS. CD80 in immune suppression by mouse ovarian carcinoma-associated Gr-1+CD11b+ myeloid cells. Cancer Res. 66(13), 6807-6815 (2006).

44. Serafini P, Mgebroff S, Noonan K, Borrello I. Myeloid-derived suppressor cells promote cross-tolerance in B-cell lymphoma by expanding regulatory T cells. Cancer Res. 68(13), 5439-5449 (2008).

45. Hipp MM, Hilf N, Walter $S$ et al. Sorafenib, but not sunitinib, affects function of dendritic cells and induction of primary immune responses. Blood 111(12), 5610-5620 (2008).

46. Finke JH, Rini B, Ireland J et al. Sunitinib reverses type-1 immune suppression and decreases T-regulatory cells in renal cell carcinoma patients. Clin. Cancer Res. Off. J. Am. Assoc. Cancer Res. 14(20), 6674-6682 (2008).

47. Fleming V, Hu X, Weber R et al. Targeting myeloid-derived suppressor cells to bypass tumor-induced immunosuppression. Front. Immunol. 9, 398 (2018).

48. Lindau D, Gielen P, Kroesen M, Wesseling P, Adema GJ. The immunosuppressive tumour network: myeloid-derived suppressor cells, regulatory T cells and natural killer T cells. Immunology 138(2), 105-115 (2013).

49. Noman MZ, Desantis G, Janji B et al. PD-L1 is a novel direct target of HIF-1 $\alpha$, and its blockade under hypoxia enhanced MDSC-mediated T cell activation. J. Exp. Med. 211(5), 781-790 (2014). 
50. Pico de Coaña Y, Masucci G, Hansson J, Kiessling R. Myeloid-derived suppressor cells and their role in CTLA-4 blockade therapy. Cancer Immunol. Immunother. CII. 63(9), 977-983 (2014).

51. Weber R, Fleming V, Hu X et al. Myeloid-derived suppressor cells hinder the anti-cancer activity of immune checkpoint inhibitors. Front. Immunol. 9, 1310 (2018).

52. Shirota $\mathrm{Y}$, Shirota $\mathrm{H}, \mathrm{Klinman} \mathrm{DM}$. Intratumoral injection of $\mathrm{CpG}$ oligonucleotides induces the differentiation and reduces the immunosuppressive activity of myeloid-derived suppressor cells. J. Immunol. Baltim. Md 1950. 188(4), 1592-1599 (2012).

53. Schaaf MB, Garg AD, Agostinis P. Defining the role of the tumor vasculature in antitumor immunity and immunotherapy. Cell Death Dis. $9(2), 115$ (2018).

54. Carmeliet P, Jain RK. Molecular mechanisms and clinical applications of angiogenesis. Nature 473(7347), 298-307 (2011).

55. Kim CG, Jang M, Kim Y et al. VEGF-A drives TOX-dependent T cell exhaustion in anti-PD-1-resistant microsatellite stable colorectal cancers. Sci. Immunol. 4(41), (2019).

56. Jain RK. Normalization of tumor vasculature: an emerging concept in antiangiogenic therapy. Science 307(5706), 58-62 (2005).

57. Rini BI, Stenzl A, Zdrojowy R et al. IMA901, a multipeptide cancer vaccine, plus sunitinib versus sunitinib alone, as first-line therapy for advanced or metastatic renal cell carcinoma (IMPRINT): a multicentre, open-label, randomised, controlled, Phase 3 trial. Lancet Oncol. 17(11), 1599-1611 (2016).

58. Amin A, Dudek AZ, Logan TF et al. Survival with AGS-003, an autologous dendritic cell-based immunotherapy, in combination with sunitinib in unfavorable risk patients with advanced renal cell carcinoma (RCC): Phase 2 study results. J. Immunother. Cancer 3, 14 (2015).

59. Mougel A, Terme M, Tanchot $\mathrm{C}$. Therapeutic cancer vaccine and combinations with antiangiogenic therapies and immune checkpoint blockade. Front. Immunol. 10, 467 (2019).

60. Tyzzer EE. Tumor immunity. Cancer Res. 1(2), 125-156 (1916).

61. Hilf N, Kuttruff-Coqui S, Frenzel K et al. Actively personalized vaccination trial for newly diagnosed glioblastoma. Nature 565(7738), 240-245 (2019).

62. Keskin DB, Anandappa AJ, Sun J et al. Neoantigen vaccine generates intratumoral T cell responses in Phase Ib glioblastoma trial. Nature 565(7738), 234-239 (2019).

63. Ott PA, Hu Z, Keskin DB et al. An immunogenic personal neoantigen vaccine for patients with melanoma. Nature 547(7662), 217-221 (2017).

64. Sahin U, Derhovanessian E, Miller M et al. Personalized RNA mutanome vaccines mobilize poly-specific therapeutic immunity against cancer. Nature 547(7662), 222-226 (2017).

65. Carreno BM, Magrini V, Becker-Hapak M et al. Cancer immunotherapy. A dendritic cell vaccine increases the breadth and diversity of melanoma neoantigen-specific T cells. Science 348(6236), 803-808 (2015).

66. Guo Y, Lei K, Tang L. Neoantigen vaccine delivery for personalized anticancer immunotherapy. Front. Immunol. 9 , 1499 (2018).

67. Hollingsworth RE, Jansen K. Turning the corner on therapeutic cancer vaccines. NPJ Vaccines. 4, 7 (2019).

68. The problem with neoantigen prediction. Nat. Biotechnol. 35(2), 97 (2017).

69. Garcia-Garijo A, Fajardo CA, Gros A. Determinants for neoantigen identification. Front. Immunol. 10, 1392 (2019).

70. Türeci O, Vormehr M, Diken M, Kreiter S, Huber C, Sahin U. Targeting the heterogeneity of cancer with individualized neoepitope vaccines. Clin. Cancer Res. Off. J. Am. Assoc. Cancer Res. 22(8), 1885-1896 (2016).

71. Chaft JE, Litvak A, Arcila ME et al. Phase II study of the GI-4000 KRAS vaccine after curative therapy in patients with stage I-III lung adenocarcinoma harboring a KRAS G12C, G12D, or G12V mutation. Clin. Lung Cancer. 15(6), 405-410 (2014).

72. Sampson JH, Archer GE, Mitchell DA et al. An epidermal growth factor receptor variant III-targeted vaccine is safe and immunogenic in patients with glioblastoma multiforme. Mol. Cancer Ther. 8(10), 2773-2779 (2009).

73. Rahma OE, Hamilton JM, Wojtowicz $\mathrm{M}$ et al. The immunological and clinical effects of mutated ras peptide vaccine in combination with IL-2, GM-CSF, or both in patients with solid tumors. J. Transl. Med. 12, 55 (2014).

74. Schuster J, Lai RK, Recht LD et al. A Phase II, multicenter trial of rindopepimut (CDX-110) in newly diagnosed glioblastoma: the ACT III study. Neuro-Oncol. 17(6), 854-861 (2015).

75. Castle JC, Uduman M, Pabla S, Stein RB, Buell JS. Mutation-derived neoantigens for cancer immunotherapy. Front. Immunol. 10, 1856 (2019).

76. Chen F, Zou Z, Du J et al. Neoantigen identification strategies enable personalized immunotherapy in refractory solid tumors. J. Clin. Invest. 129(5), 2056-2070 (2019).

77. Verdegaal EME, de Miranda NFCC, Visser M et al. Neoantigen landscape dynamics during human melanoma-T cell interactions. Nature 536(7614), 91-95 (2016). 
78. Oxnard GR, Arcila ME, Chmielecki J, Ladanyi M, Miller VA, Pao W. New strategies in overcoming acquired resistance to epidermal growth factor receptor tyrosine kinase inhibitors in lung cancer. Clin. Cancer Res. Off. J. Am. Assoc. Cancer Res. 17(17), 5530-5537 (2011).

79. Toy W, Shen Y, Won H et al. ESR1 ligand-binding domain mutations in hormone-resistant breast cancer. Nat. Genet. 45(12), 1439-1445 (2013).

80. Jeselsohn R, Yelensky R, Buchwalter G et al. Emergence of constitutively active estrogen receptor- $\alpha$ mutations in pretreated advanced estrogen receptor-positive breast cancer. Clin. Cancer Res. Off. J. Am. Assoc. Cancer Res. 20(7), 1757-1767 (2014).

81. United States Patent Application: 0180326030 (2019). http://appft.uspto.gov/netacgi/nph-Parser?Sect1=PTO1\&Sect2=HITOFF\&p=1 $\& u=/$ netahtml/PTO/srchnum.html\&r=1\&f=G\&l=50\&d=PG01\&s1=20180326030.PGNR

82. Mukai $\mathrm{K}$, Yasutomi $\mathrm{Y}$, Watanabe $\mathrm{M}$ et al. HER2 peptide-specific $\mathrm{CD} 8^{+} \mathrm{T}$ cells are proportionally detectable long after multiple DNA vaccinations. Gene Ther. 9(13), 879-888 (2002).

83. Mittendorf EA, Alatrash G, Qiao N et al. Breast cancer cell uptake of the inflammatory mediator neutrophil elastase triggers an anticancer adaptive immune response. Cancer Res. 72(13), 3153-3162 (2012).

84. Mittendorf EA, Storrer CE, Foley RJ et al. Evaluation of the HER2/neu-derived peptide GP2 for use in a peptide-based breast cancer vaccine trial. Cancer. 106(11), 2309-2317 (2006).

85. Peoples GE, Gurney JM, Hueman MT et al. Clinical trial results of a HER2/neu (E75) vaccine to prevent recurrence in high-risk breast cancer patients. J. Clin. Oncol. Off. J. Am. Soc. Clin. Oncol. 23(30), 7536-7545 (2005).

86. Melief CJM, van der Burg SH. Immunotherapy of established (pre)malignant disease by synthetic long peptide vaccines. Nat. Rev Cancer. 8(5), 351-360 (2008).

87. Datta J, Fracol M, McMillan MT et al. Association of depressed anti-HER2 T-Helper Type 1 response with recurrence in patients with completely treated HER2-Positive breast cancer: role for immune monitoring. JAMA Oncol. 2(2), 242 (2016).

88. Crosby EJ, Gwin W, Blackwell K et al. Vaccine-induced Memory CD8 ${ }^{+} \mathrm{T}$ cells provide clinical benefit in HER2 expressing breast cancer: a mouse to human translational study. Clin. Cancer Res. 25(9), 2725-2736 (2019).

- Demonstrates that, with an immunogenic virally-derived platform, immunization with a tumor-associated antigen (HER2) drives strong $\mathrm{CD8}^{+} \mathrm{T}$ cell effector and memory responses with a coincident reduction in Tregs.

89. Costa R, Shah AN, Santa-Maria CA et al. Targeting epidermal growth factor receptor in triple negative breast cancer: new discoveries and practical insights for drug development. Cancer Treat. Rev. 53, 111-119 (2017).

90. Witkiewicz AK, Knudsen KE, Dicker AP, Knudsen ES. The meaning of p16(ink4a) expression in tumors: functional significance, clinical associations and future developments. Cell Cycle Georget. Tex. 10(15), 2497-2503 (2011).

91. Finlay CA, Hinds PW, Levine AJ. The $p 53$ proto-oncogene can act as a suppressor of transformation. Cell. 57(7), 1083-1093 (1989).

92. Levine AJ. p53, the cellular gatekeeper for growth and division. Cell. 88(3), 323-331 (1997).

93. Wiedenfeld EA, Fernandez-Viña M, Berzofsky JA, Carbone DP. Evidence for selection against human lung cancers bearing p 53 missense mutations which occur within the HLA A*0201 peptide consensus motif. Cancer Res. 54(5), 1175-1177 (1994).

94. van der Burg SH, de Cock K, Menon AG et al. Long lasting p53-specific T cell memory responses in the absence of anti-p 53 antibodies in patients with resected primary colorectal cancer. Eur. J. Immunol. 31(1), 146-155 (2001).

95. Leffers N, Lambeck AJA, Gooden MJM et al. Immunization with a p53 synthetic long peptide vaccine induces p53-specific immune responses in ovarian cancer patients, a Phase II trial. Int. J. Cancer. 125(9), 2104-2113 (2009).

96. Antonia SJ. Combination of p53 cancer vaccine with chemotherapy in patients with extensive stage small cell lung cancer. Clin. Cancer Res. 12(3), 878-887 (2006).

97. Hardwick NR, Frankel P, Ruel C et al. p53-reactive T cells are associated with clinical benefit in patients with platinum-resistant epithelial ovarian cancer after treatment with a p53 vaccine and gemcitabine chemotherapy. Clin. Cancer Res. 24(6), 1315-1325 (2018).

98. Zeestraten ECM, Speetjens FM, Welters MJP et al. Addition of interferon- $\alpha$ to the $\mathrm{p} 53-\mathrm{SLP}^{\circledR}{ }^{\circledR}$ vaccine results in increased production of interferon- $\gamma$ in vaccinated colorectal cancer patients: a Phase I/II clinical trial. Int. J. Cancer. 132(7), 1581-1591 (2013).

99. Kiaris H, Chatzistamou I, Trimis G, Frangou-Plemmenou M, Pafiti-Kondi A, Kalofoutis A. Evidence for nonautonomous effect of p53 tumor suppressor in carcinogenesis. Cancer Res. 65(5), 1627-1630 (2005).

100. Guo C, Manjili MH, Subjeck JR, Sarkar D, Fisher PB, Wang X-Y. Therapeutic cancer vaccines: past, present, and future. Adv. Cancer Res. 119, 421-475 (2013).

101. Samuels Y, Waldman T. Oncogenic mutations of PIK3CA in human cancers. Curr. Top. Microbiol. Immunol. 347, 21-41 (2010).

102. Baker AT, Mundy RM, Davies JA, Rizkallah PJ, Parker AL. Human adenovirus type 26 uses sialic acid-bearing glycans as a primary cell entry receptor. Sci. Adv. 5(9), eaax3567 (2019).

103. Altenburg AF, van de Sandt CE, Li BWS et al. Modified vaccinia virus ankara preferentially targets antigen presenting cells in vitro, ex vivo and in vivo. Sci. Rep. 7(1), 8580 (2017).

104. MacDonald GH, Johnston RE. Role of dendritic cell targeting in Venezuelan equine encephalitis virus pathogenesis. J. Virol. 74(2), 914-922 (2000). 
105. Coughlan L, Alba R, Parker AL et al. Tropism-modification strategies for targeted gene delivery using adenoviral vectors. Viruses. 2(10), 2290-2355 (2010).

106. Reichmuth AM, Oberli MA, Jaklenec A, Langer R, Blankschtein D. mRNA vaccine delivery using lipid nanoparticles. Ther. Deliv. 7(5), 319-334 (2016).

107. Geall AJ, Verma A, Otten GR et al. Nonviral delivery of self-amplifying RNA vaccines. Proc. Natl Acad. Sci. USA 109(36), 14604-14609 (2012)

108. Kranz LM, Diken M, Haas H et al. Systemic RNA delivery to dendritic cells exploits antiviral defence for cancer immunotherapy. Nature 534(7607), 396-401 (2016).

109. Johansson DX, Ljungberg K, Kakoulidou M, Liljeström P. Intradermal electroporation of naked replicon RNA elicits strong immune responses. PloS ONE 7(1), e29732 (2012).

110. Pardi N, Hogan MJ, Naradikian MS et al. Nucleoside-modified mRNA vaccines induce potent $T$ follicular helper and germinal center $B$ cell responses. J. Exp. Med. 215(6), 1571-1588 (2018).

111. Calabro S, Tortoli M, Baudner BC et al. Vaccine adjuvants alum and MF59 induce rapid recruitment of neutrophils and monocytes that participate in antigen transport to draining lymph nodes. Vaccine 29(9), 1812-1823 (2011).

112. Langlet C, Tamoutounour S, Henri S et al. CD64 expression distinguishes monocyte-derived and conventional dendritic cells and reveals their distinct role during intramuscular immunization. J. Immunol. Baltim. Md 1950. 188(4), 1751-1760 (2012).

113. Lindsay KE, Bhosle SM, Zurla C et al. Visualization of early events in mRNA vaccine delivery in non-human primates via PET-CT and near-infrared imaging. Nat. Biomed. Eng. 3(5), 371-380 (2019).

- Shows how the spatiotemporal trafficking of vaccines in Nonhuman primates could impact subsequent development of immunological memory.

114. Karikó K, Muramatsu H, Ludwig J, Weissman D. Generating the optimal mRNA for therapy: HPLC purification eliminates immune activation and improves translation of nucleoside-modified, protein-encoding mRNA. Nucleic Acids Res. 39(21), e142 (2011).

115. Ventoso I, Sanz MA, Molina S, Berlanga JJ, Carrasco L, Esteban M. Translational resistance of late alphavirus mRNA to eIF2alpha phosphorylation: a strategy to overcome the antiviral effect of protein kinase PKR. Genes Dev. 20(1), 87-100 (2006).

116. Näslund TI, Kostic L, Nordström EK, Chen M, Liljeström P. Role of innate signalling pathways in the immunogenicity of alphaviral replicon-based vaccines. Virol. J. 8, 36 (2011).

117. Atkins GJ. The pathogenesis of alphaviruses. ISRN Virol. 2013, 1-22 (2013).

118. De Beuckelaer A, Pollard C, Van Lint S et al. Type I Interferons interfere with the capacity of mRNA lipoplex vaccines to elicit cytolytic T cell responses. Mol. Ther. J. Am. Soc. Gene Ther. 24(11), 2012-2020 (2016).

119. De Beuckelaer A, Grooten J, De Koker S. Type I interferons modulate CD8 ${ }^{+}$T cell immunity to mRNA vaccines. Trends Mol. Med. 23(3), 216-226 (2017).

120. Pepini T, Pulichino A-M, Carsillo T et al. Induction of an IFN-mediated antiviral response by a self-amplifying RNA vaccine: implications for vaccine design. J. Immunol. Baltim. Md 1950. 198(10), 4012-4024 (2017).

121. Khong H, Overwijk WW. Adjuvants for peptide-based cancer vaccines. J. Immunother. Cancer. 4(1), 56 (2016).

122. Seubert A, Monaci E, Pizza M, O'Hagan DT, Wack A. The adjuvants aluminum hydroxide and MF59 induce monocyte and granulocyte chemoattractants and enhance monocyte differentiation toward dendritic cells. J. Immunol. Baltim. Md 1950. 180(8), 5402-5412 (2008).

123. van Aalst S, Ludwig IS, van Kooten PJS, van der Zee R, van Eden W, Broere F. Dynamics of APC recruitment at the site of injection following injection of vaccine adjuvants. Vaccine 35(12), 1622-1629 (2017).

124. Lugo-Villarino G, Maldonado-Lopez R, Possemato R, Penaranda C, Glimcher LH. T-bet is required for optimal production of IFN-and antigen-specific T cell activation by dendritic cells. Proc. Natl. Acad. Sci. 100(13), 7749-7754 (2003).

125. Pantel A, Teixeira A, Haddad E, Wood EG, Steinman RM, Longhi MP. Direct type I IFN but not MDA5/TLR3 activation of dendritic cells is required for maturation and metabolic shift to glycolysis after poly IC stimulation. PLoS Biol. 12(1), e1001759 (2014).

126. Widera G, Austin M, Rabussay D et al. Increased DNA vaccine delivery and immunogenicity by electroporation in vivo. J. Immunol. 164(9), 4635-4640 (2000).

127. Vogel AB, Lambert L, Kinnear $\mathrm{E}$ et al. Self-amplifying RNA vaccines give equivalent protection against influenza to mRNA vaccines but at much lower doses. Mol. Ther. 26(2), 446-455 (2018).

128. Sawicki DL, Silverman RH, Williams BR, Sawicki SG. Alphavirus minus-strand synthesis and persistence in mouse embryo fibroblasts derived from mice lacking RNase L and protein kinase R. J. Virol. 77(3), 1801-1811 (2003).

129. Tosello V, Zamarchi R, Merlo A et al. Differential expression of constitutive and inducible proteasome subunits in human monocyte-derived DC differentiated in the presence of IFN-alpha or IL-4. Eur. J. Immunol. 39(1), 56-66 (2009).

130. Shin E-C, Seifert U, Kato T et al. Virus-induced type I IFN stimulates generation of immunoproteasomes at the site of infection. J. Clin. Invest. 116(11), 3006-3014 (2006). 
131. Barouch DH, Tomaka FL, Wegmann F et al. Evaluation of a mosaic HIV-1 vaccine in a multicentre, randomised, double-blind, placebo-controlled, Phase 1/2a clinical trial (APPROACH) and in rhesus monkeys (NH 13-19). The Lancet. 392(10143), 232-243 (2018).

132. Stephenson KE, Keefer MC, Bunce CA et al. First-in-human randomized controlled trial of an oral, replicating adenovirus 26 vector vaccine for HIV-1. PLoS ONE 13(11), e0205139 (2018).

133. Ledgerwood JE, DeZure AD, Stanley DA et al. Chimpanzee adenovirus vector ebola vaccine. N. Engl. J. Med. 376(10), 928-938 (2017).

134. Geisbert TW, Bailey M, Hensley L et al. Recombinant adenovirus serotype 26 (Ad26) and Ad35 vaccine vectors bypass immunity to Ad5 and protect nonhuman primates against ebolavirus challenge. J. Virol. 85(9), 4222-4233 (2011).

135. Venkatraman N, Ndiaye BP, Bowyer G et al. Safety and immunogenicity of a heterologous prime-boost ebola virus vaccine regimen in healthy adults in the United Kingdom and senegal. J. Infect. Dis. 219(8), 1187-1197 (2019).

136. Hill AVS, Reyes-Sandoval A, O’Hara G et al. Prime-boost vectored malaria vaccines: progress and prospects. Hum. Vaccin. 6(1), 78-83 (2010).

137. Mogler MA, Kamrud KI. RNA-based viral vectors. Expert Rev. Vaccines 14(2), 283-312 (2015).

138. Lundstrom K. Alphavirus-based vaccines. Viruses 6(6), 2392-2415 (2014).

139. Gothelf A, Gehl J. What you always needed to know about electroporation based DNA vaccines. Hum. Vaccines Immunother. 8(11), 1694-1702 (2012).

140. Gaudinski MR, Houser KV, Morabito KM et al. Safety, tolerability, and immunogenicity of two Zika virus DNA vaccine candidates in healthy adults: randomised, open-label, phase 1 clinical trials. The Lancet 391(10120), 552-562 (2018).

141. Ulmer JB, Mansoura MK, Geall AJ. Vaccines 'on demand': science fiction or a future reality. Expert Opin. Drug Discov. 10(2), 101-106 (2015).

142. Knudsen ML, Ljungberg K, Kakoulidou $\mathrm{M}$ et al. Kinetic and phenotypic analysis of CD8 ${ }^{+} \mathrm{T}$ cell responses after priming with alphavirus replicons and homologous or heterologous booster immunizations. J. Virol. 88(21), 12438-12451 (2014).

143. Lutz J, Lazzaro S, Habbeddine $\mathrm{M}$ et al. Unmodified mRNA in LNPs constitutes a competitive technology for prophylactic vaccines. NPJ Vaccines. 2, 29 (2017).

144. Feldman RA, Fuhr R, Smolenov I et al. mRNA vaccines against H10N8 and H7N9 influenza viruses of pandemic potential are immunogenic and well tolerated in healthy adults in Phase 1 randomized clinical trials. Vaccine 37(25), 3326-3334 (2019).

145. Purcell AW, McCluskey J, Rossjohn J. More than one reason to rethink the use of peptides in vaccine design. Nat. Rev. Drug Discov. 6(5), 404-414 (2007).

146. Li W, Joshi MD, Singhania S, Ramsey KH, Murthy AK. Peptide vaccine: progress and challenges. Vaccines (Basel) 2(3), 515-536 (2014).

147. Abelin JG, Harjanto D, Malloy M et al. Defining HLA-II ligand processing and binding rules with mass spectrometry enhances cancer epitope prediction. Immunity 51(4), 766-779.e17 (2019).

148. Mertens M, Singh JA. Anakinra for rheumatoid arthritis. Cochrane Database Syst. Rev. (1), CD005121 (2009).

149. Smolen JS, Aletaha D, Choy EH, Jones SA, Takeuchi T, McInnes I. Targeting IL-6: a review of data. Consid. Med. 2(1), 12-18 (2018).

150. Zhao J, Cao Y, Lei Z, Yang Z, Zhang B, Huang B. Selective depletion of CD4+CD25+Foxp3+ regulatory T cells by low-dose cyclophosphamide is explained by reduced intracellular ATP levels. Cancer Res. 70(12), 4850-4858 (2010).

151. Scheper W, Kelderman S, Fanchi LF et al. Low and variable tumor reactivity of the intratumoral TCR repertoire in human cancers. Nat. Med. 25(1), 89-94 (2019).

-• Describes the fact that many $\mathrm{T}$ cells present in the tumor microenvironment do not express tumor reactive $\mathrm{T}$ cell receptors, suggesting that reawakening these $\mathrm{T}$ cells with checkpoint inhibitors will not be sufficient and priming de novo $\mathrm{T}$-cell responses is critical.

152. Robert L, Tsoi J, Wang X et al. CTLA4 blockade broadens the peripheral T-cell receptor repertoire. Clin. Cancer Res. 20(9), 2424-2432 (2014).

153. Postow MA, Manuel M, Wong P et al. Peripheral T cell receptor diversity is associated with clinical outcomes following ipilimumab treatment in metastatic melanoma. J. Immunother. Cancer. 3, 23 (2015).

154. Hopkins AC, Yarchoan M, Durham JN et al. T cell receptor repertoire features associated with survival in immunotherapy-treated pancreatic ductal adenocarcinoma. JCI Insight. 3(13), e122092 (2018).

155. Hildner K, Edelson BT, Purtha WE et al. Batf3 deficiency reveals a critical role for CD8alpha+ dendritic cells in cytotoxic T cell immunity. Science. 322(5904), 1097-1100 (2008).

156. Grajales-Reyes GE, Iwata A, Albring J et al. Batf3 maintains autoactivation of Irf8 for commitment of a CD8 $\alpha(+)$ conventional DC clonogenic progenitor. Nat. Immunol. 16(7), 708-717 (2015).

157. Sánchez-Paulete AR, Teijeira Á, Quetglas JI et al. Intratumoral immunotherapy with XCL1 and sFlt3L encoded in recombinant semliki forest virus-derived vectors fosters dendritic cell-mediated T-cell cross-priming. Cancer Res. 78(23), 6643-6654 (2018). 
158. Poulin LF, Salio M, Griessinger E et al. Characterization of human DNGR-1 + BDCA3 ${ }^{+}$leukocytes as putative equivalents of mouse CD8 $\alpha^{+}$dendritic cells. J. Exp. Med. 207(6), 1261-1271 (2010).

159. Wilson EB, Yamada DH, Elsaesser $\mathrm{H}$ et al. Blockade of chronic type I interferon signaling to control persistent LCMV infection. Science 340(6129), 202-207 (2013).

160. Teijaro JR, Ng C, Lee AM et al. Persistent LCMV infection is controlled by blockade of type I interferon signaling. Science 340(6129), 207-211 (2013).

161. Aris M, Mordoh J, Barrio MM. Immunomodulatory monoclonal antibodies in combined immunotherapy trials for cutaneous melanoma. Front. Immunol. 8, 1024 (2017).

162. Chung V, Kos FJ, Hardwick N et al. Evaluation of safety and efficacy of p53MVA vaccine combined with pembrolizumab in patients with advanced solid cancers. Clin. Transl. Oncol. Off. Publ. Fed. Span. Oncol. Soc. Natl. Cancer Inst. Mex. 21(3), 363-372 (2019).

163. Gibney GT, Kudchadkar RR, DeConti RC et al. Safety, correlative markers, and clinical results of adjuvant nivolumab in combination with vaccine in resected high-risk metastatic melanoma. Clin. Cancer Res. Off. J. Am. Assoc. Cancer Res. 21(4), 712-720 (2015).

164. Massarelli E, William W, Johnson F et al. Combining immune checkpoint blockade and tumor-specific vaccine for patients with incurable human papillomavirus 16-related cancer: a Phase 2 clinical Trial. JAMA Oncol. 5(1), 67-73 (2019).

165. Verma V, Shrimali RK, Ahmad S et al. PD-1 blockade in subprimed CD8 cells induces dysfunctional PD- $1^{+}$CD $38^{\text {hi }}$ cells and anti-PD-1 resistance. Nat. Immunol. 20(9), 1231-1243 (2019).

-• Highlights an important point that incorrect staging of checkpoint inhibitors is detrimental to the antitumor response if delivered during T-cell priming.

166. Im SJ, Hashimoto M, Gerner MY et al. Defining $C D 8^{+} \mathrm{T}$ cells that provide the proliferative burst after PD-1 therapy. Nature. 537(7620), 417-421 (2016).

167. Leong YA, Chen Y, Ong HS et al. CXCR5(+) follicular cytotoxic T cells control viral infection in B cell follicles. Nat. Immunol. 17(10), 1187-1196 (2016).

168. Wu T, Ji Y, Moseman EA et al. The TCF1-Bcl6 axis counteracts type I interferon to repress exhaustion and maintain T cell stemness. Sci. Immunol. 1(6), pii: eaai8593 (2016).

169. Huang Z, Zak J, Pratumchai I et al. IL-27 promotes the expansion of self-renewing CD8 ${ }^{+} \mathrm{T}$ cells in persistent viral infection. J. Exp. Med. 216(8), 1791-1808 (2019).

170. Seidel JA, Otsuka A, Kabashima K. Anti-PD-1 and anti-CTLA-4 therapies in cancer: mechanisms of action, efficacy, and limitations. Front. Oncol. 8, 86 (2018).

171. Lines JL, Sempere LF, Broughton T, Wang L, Noelle R. VISTA is a novel broad-spectrum negative checkpoint regulator for cancer immunotherapy. Cancer Immunol. Res. 2(6), 510-517 (2014).

172. Chew GM, Fujita T, Webb GM et al. TIGIT marks exhausted T cells, correlates with disease progression, and serves as a target for immune restoration in HIV and SIV infection. PLoS Pathog. 12(1), e1005349 (2016).

173. Marin-Acevedo JA, Dholaria B, Soyano AE, Knutson KL, Chumsri S, Lou Y. Next generation of immune checkpoint therapy in cancer: new developments and challenges. J. Hematol. Oncol. J Hematol. Oncol. 11(1), 39 (2018). 